\title{
Resolved Kinematics of Runaway and Field OB Stars in the Small Magellanic Cloud
}

\author{
M. S. Oey ${ }^{1}$ (10) J. Dorigo Jones ${ }^{1}$, N. Castro ${ }^{1,2}$ (1) , P. Zivick ${ }^{3}$ (1), G. Besla ${ }^{4}$, H. C. Januszewski ${ }^{1,5}$, \\ M. Moe ${ }^{4}$, N. Kallivayalil ${ }^{3}$ (D), and D. J. Lennon ${ }^{6,7}$ (iD \\ ${ }^{1}$ Department of Astronomy University of Michigan 1085 South University Avenue, Ann Arbor, MI 48109-1107, USA; msoey@umich.edu \\ ${ }^{2}$ Present address: Leibniz-Institut für Astrophysik An der Sternwarte 16 D-14482 Potsdam, Germany \\ ${ }^{3}$ Department of Astronomy University of Virginia 530 McCormick Road, Charlottesville, VA 22904, USA \\ ${ }^{4}$ Steward Observatory University of Arizona 933 North Cherry Avenue, Tucson, AZ 85721, USA \\ ${ }^{5}$ Present address: Gemini Observatory 670 North A'ohoku Place, Hilo, HI 96720, USA \\ ${ }^{6}$ ESA, European Space Astronomy Centre Apdo. de Correos 78 E-28691 Villanueva de la Cañada, Madrid, Spain \\ 7 Instituto de Astrofísica de Canarias E-38205 La Laguna, Tenerife, Spain \\ Received 2018 September 6; revised 2018 October 11; accepted 2018 October 13; published 2018 October 26
}

\begin{abstract}
We use Gaia Data Release 2 proper motions of field OB stars from the Runaways and Isolated O-Type Star Spectroscopic Survey of the Small Magellanic Cloud (SMC) to study the kinematics of runaway stars. The data reveal that the SMC Wing has a systemic peculiar motion relative to the SMC Bar of $\left(v_{\alpha}, v_{\delta}\right)=$ $(62 \pm 7,-18 \pm 5) \mathrm{km} \mathrm{s}^{-1}$ and relative radial velocity $+4.5 \pm 5.0 \mathrm{~km} \mathrm{~s}^{-1}$. This unambiguously demonstrates that these two regions are kinematically distinct: the Wing is moving away from the Bar, and towards the Large Magellanic Cloud with a 3D velocity of $64 \pm 10 \mathrm{~km} \mathrm{~s}^{-1}$. This is consistent with models for a recent, direct collision between the Clouds. We present transverse velocity distributions for our field OB stars, confirming that unbound runaways comprise on the order of half our sample, possibly more. Using eclipsing binaries and doublelined spectroscopic binaries as tracers of dynamically ejected runaways, and high-mass X-ray binaries (HMXBs) as tracers of runaways accelerated by supernova kicks, we find significant contributions from both populations. The data suggest that HMXBs have lower velocity dispersion relative to dynamically ejected binaries, consistent with the former group corresponding to less energetic supernova kicks that failed to unbind the components. Evidence suggests that our fast runaways are dominated by dynamical, rather than supernova, ejections.
\end{abstract}

Key words: binaries: general - galaxies: star clusters: general - Magellanic Clouds - stars: kinematics and dynamics - stars: massive $-\mathrm{X}$-rays: binaries

Supporting material: machine-readable table

\section{Introduction: Runaway OB Stars}

Field OB stars constitute a significant subset of the massive star population in star-forming galaxies. Given the power-law cluster mass distribution, Oey et al. (2004) showed that field OB stars typically comprise $20 \%-30 \%$ of massive stars. However, the field additionally includes significant numbers of high-velocity runaway stars ejected from clusters (Blaauw 1961; Hoogerwerf et al. 2000). The classic work by Blaauw (1961) found that about $20 \%$ of early B stars and 3\% of O stars are runaways, and Moffat et al. (1998) found a runaway fraction of $\mathrm{O}$ and Wolf-Rayet stars of $14 \%$ from HIPPARCOS space velocities. However, infrared work by de Wit et al. (2005) suggests that over $90 \%$ of O stars are runaways, and some studies suggest that all truly isolated field massive stars are runaways (e.g., Pflamm-Altenburg \& Kroupa 2010; Gvaramadze et al. 2011). On the other hand, a variety of observational evidence suggests that field objects formed in relative isolation are also a major, if not dominant, component of the field massive star population (e.g., Oey et al. 2013; Lamb et al. 2016).

Two principal mechanisms are responsible for generating runaway stars. One is dynamical ejection from gravitationally unstable configurations (Poveda et al. 1967; Leonard \& Duncan 1988); another is the acceleration of a star when its binary companion explodes as a supernova (SN; Blaauw 1961). These are dominated, for higher velocity runaways, by explosions that generate a recoil "kick" to the companion, rather than simple "slingshot" acceleration (e.g., Renzo et al. 2018).
The relative importance of the dynamical versus $\mathrm{SN}$ mechanisms is poorly known. For the latter, a minority of runaways should retain their neutron star companions, while SNe disrupt most of these binaries (e.g., Brandt \& Podsiadlowski 1995; Renzo et al. 2018). This is supported by searches for runaways with neutron star companions (e.g., Philp et al. 1996; Sayer et al. 1996).

The dynamical ejection mechanism takes place primarily via binary-binary interactions (Poveda et al. 1967; Leonard \& Duncan 1988). This is the only process that can yield binary runaways consisting of two non-compact stars, in addition to single runaways. From 42 runaways in the Galactic field $\mathrm{O}$ star sample, the frequency of non-compact, multiple runaways is at least $\sim 15 \%$ of O star runaways, based on detections of doublelined spectroscopic binaries (SB2) and astrometric binaries (Mason et al. 2009). If such a value is confirmed, dynamical ejection may well dominate the massive runaway population. However, dynamical ejection may require unusual mass ratios and orbital parameters (Leonard \& Duncan 1990). For example, for a binary-binary ejection model, Clarke \& Pringle (1992) require nearly all $O$ stars to have close binary companions with mass ratios $>0.25$, to achieve a runaway fraction $>10 \%$. Although most $\mathrm{O}$ stars indeed have close $\mathrm{OB}$ companions (Sana et al. 2012), there is a significant contribution from lower-mass close companions as well (Moe \& Di Stefano 2015).

Hence, the statistical properties and fundamental parameters of the OB runaway population offer critical diagnostics of the 
ejection mechanisms, and their statistics also depend strongly on cluster properties and dynamical evolution (e.g., Poveda et al. 1967; Hills 1980). Evaluating the frequency and properties of runaways is therefore vital to understanding the nature of both the field population and clusters (e.g., Clarke \& Pringle 1992; Portegies Zwart 2000). However, testing such predictions has been limited to date by the substantial uncertainties for runaway statistics and inhomogeneous data in the Milky Way.

Here, we examine the kinematics of runaway OB stars in an extragalactic environment: the Small Magellanic Cloud (SMC), where statistical completeness is easily evaluated. Gaia Data Release 2 (DR2; Gaia Collaboration et al. 2018) now offers an outstanding data set of proper motions (PMs) for the SMC, which is located at high Galactic latitude and low extinction, owing to its low metallicity. Our study is based on the Runaways and Isolated O-Type Star Spectroscopic Survey of the SMC (RIOTS4), which is yielding a detailed, quantitative characterization of this field $\mathrm{OB}$ population (e.g., Lamb et al. 2016). This sample also offers an opportunity to look at large-scale stellar kinematics of the SMC's young population.

\section{RIOTS4 Proper Motions from Gaia}

The RIOTS4 field star sample is defined from Oey et al. (2004), who used the $U B V R$ photometric survey of the SMC by Massey (2002) to identify OB-star candidates based on having reddening-free parameter $Q_{\mathrm{UBR}} \leqslant-0.84$ and $B \leqslant 15.21$. These serve as uniform selection criteria for stars earlier than spectral type $\sim$ B0.5. Field and cluster stars were defined using the friends-of-friends algorithm of Battinelli (1991), adopting a clustering length of $28 \mathrm{pc}$, which yields 374 field stars, or $28 \%$ of all SMC OB stars identified by Oey et al. (2004). An additional 23 O stars in the RIOTS4 sample were identified using ultraviolet (UV) photometric criteria on data from the Ultraviolet Imaging Telescope, yielding a total of 397 stars. The two subsamples are given in Tables 1 and 2, respectively, of Lamb et al. (2016; T1, T2). Spectroscopic observations of these RIOTS4 stars were obtained at the Magellan Telescopes using the Inamori-Magellan Areal Camera and Spectrograph (IMACS) and the Magellan Inamori Kyocera Echelle (MIKE) spectrograph (Lamb et al. 2016), with ongoing, multi-year monitoring in the Wing region using the M2FS multi-fiber spectrograph. The latter data yield systemic radial velocities (RVs) for detected binary systems; a smaller region in the SMC Bar was similarly monitored with IMACS, and systemic RVs reported in Table 3 of Lamb et al. (2016; T3).

We identify the RIOTS4 stars in the Gaia DR2 catalog by specifying a position match within $3^{\prime \prime}$ and magnitude match $|G-V|<0.3$, yielding 328 matches. We further vet the sample by including only stars having both R.A. and decl. PM errors $<1 \sigma$ from the median error, eliminating 12 stars. We also delete one more star that has R.A. or decl. errors $>3.5 \sigma$ after the initial clip. The final R.A. and decl. standard deviations are $55 \mathrm{~km} \mathrm{~s}^{-1}$ and $37 \mathrm{~km} \mathrm{~s}^{-1}$, respectively. Our stars are generally in the range $12.5<G<15.5$. Figure 1(a) depicts the PMs for these 315 RIOTS4 stars, adopting a mean SMC distance modulus of 18.99 (Cioni et al. 2000; Table 1). The PMs are residuals relative to the SMC systemic PM of $\left(\mu_{\alpha}, \mu_{\delta}\right)=$ $(0.754,-1.252)$ mas $\mathrm{yr}^{-1}$ from Piatek et al. (2008), which is the published value that minimizes residuals in the SMC Bar. The shown vectors are also corrected for geometric perspective using the model of van der Marel et al. (2002), but adopting a center of motion at the midpoint between the H I (Stanimirović et al. 2004) and stellar (Ripepi et al. 2017) kinematic centers. Figure 1 reveals a pattern of motion consistent with that of Zivick et al. (2018). We also apply geometric corrections to the RVs and subtract the median SMC systemic RV of $152 \mathrm{~km} \mathrm{~s}^{-1}$ for the Bar.

It is apparent in Figure 1 that the SMC Wing shows a median systemic PM of $\left(\mu_{\alpha}, \mu_{\delta}\right)=(0.207 \pm 0.025,-0.060 \pm$

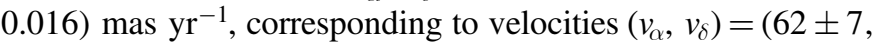
$-18 \pm 5) \mathrm{km} \mathrm{s}^{-1}$ and a total transverse velocity $v_{\perp}=64 \pm$ $8 \mathrm{~km} \mathrm{~s}^{-1}$. This Wing peculiar motion is obtained after deleting 11 stars within 0.5 of the boundary shown in Figure 1. Panel (b) shows the PMs with the 68 Wing stars corrected for this additional peculiar motion. This effect is robust to the choice of systemic PM and kinematic center. In applying geometric corrections to the RVs, we find that the RV offset reported by Lamb et al. (2016), who did not correct for perspective, is due primarily to this effect. The Wing median RV offset from our data is now $+4.5 \pm 5.0 \mathrm{~km} \mathrm{~s}^{-1}$.

Table 1 lists the total residual transverse velocities $v_{\perp}$ and $\mathrm{RV}$, along with non-residual PMs for our field OB stars. We also give locally determined transverse velocities (see Section 3.2). Stars in the Wing region (Figure 1) are indicated, and their residual values are corrected for the Wing peculiar motions in PM and RV. All PM values are based on the original SMC geometric correction described above; our models show that a specific correction for the Wing would modify the velocities by at most $3.5 \mathrm{~km} \mathrm{~s}^{-1}$, whereas systematic errors on the geometric correction are on the order of $30 \mathrm{~km} \mathrm{~s}^{-1}$. The FWHM of the Cepheid distance distribution yields a variation of $15 \%$ (e.g., Ripepi et al. 2017), with extremes up to $50 \%$, given the SMC's end-on orientation to the line of sight. These imply distance uncertainties that propagate directly to our transverse velocities.

\section{SMC Field OB Kinematics}

\subsection{Proper Motion of the SMC Wing}

While it is necessary to correct for the Wing's systemic motion to identify runaway stars, our data also clearly reveal that the Wing and Bar are kinematically distinct components, with a 3D offset of $64 \pm 10 \mathrm{~km} \mathrm{~s}^{-1}$. The Wing has been identified as the southeast component of the SMC, and extends $\gtrsim 2^{\circ}$ beyond our observed data set, merging with the Magellanic Bridge linking the SMC to the Large Magellanic Cloud (LMC). Previous work (e.g., Brück 1978; Dobbie et al. 2014) shows an older co-existing Wing stellar population having RVs similar to those of young stars. This suggests that our sample is a good tracer of the bulk motion of this region, but follow-up examination of Gaia PMs for red giant stars is needed to understand differentials between the old and young populations.

The dynamical state of the Wing provides a vital kinematic discriminant for dynamical models of the internal structure of the SMC, the recent encounter history of the Magellanic Clouds, and the formation of the Magellanic Bridge (e.g., Besla et al. 2012; Zivick et al. 2018). In particular, the Wing kinematics seen here are consistent with transverse motion along the Bridge toward the LMC, instead of perpendicular to the Bridge. This vividly confirms models for a recent, direct collision between the Clouds 100-200 Myr ago, for which gas velocities are expected to be aligned with the Bridge. In 

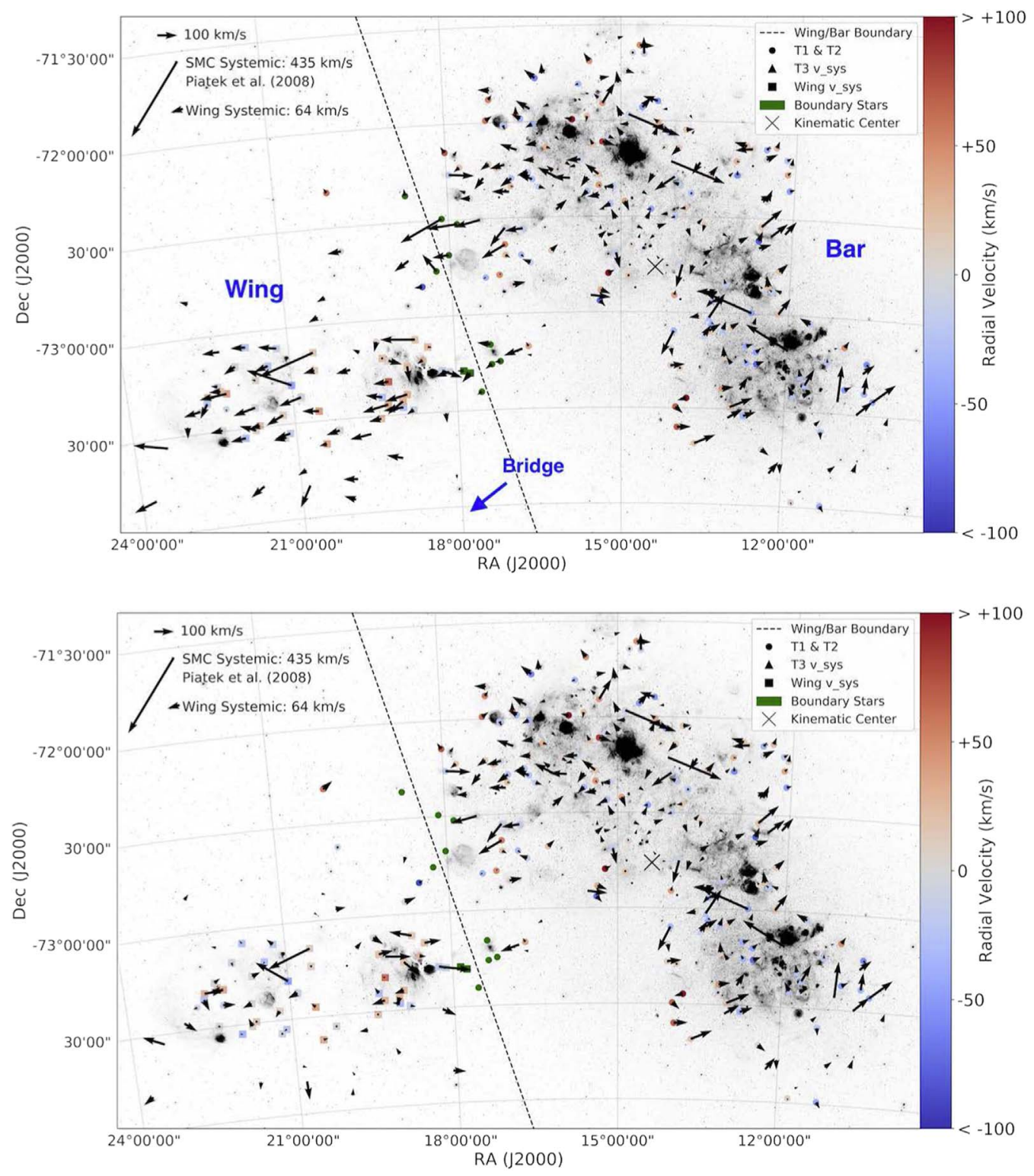

Figure 1. Vector map of 315 Gaia DR2 PM residuals for RIOTS4 field OB stars superposed on H $\alpha$ image from Smith et al. (2005). Panel (a, top) shows only corrections for geometric perspective and SMC systemic velocity. Panel (b, bottom) additionally includes a separate peculiar PM and RV correction for Wing stars. The adopted boundary between the so-called Wing and Bar regions is shown by the dashed line, with stars removed from the PM samples indicated in green. The vectors for the Wing peculiar PM correction and SMC systemic PM are shown, as is the adopted kinematic center. Colors show available RVs for 216 stars. Symbols indicate RV source, with triangles and squares showing stars with systemic RV measurements from multi-epoch monitoring; T1, T2, and T3 refer to data from tables in Lamb et al. (2016; see Section 2). The direction toward the Magellanic Bridge is indicated.

contrast, motions perpendicular to the Bridge are theoretically expected in a tidal stripping scenario of an SMC that did not collide with the LMC, allowing it to retain ordered rotation. The absence of perpendicular motion is consistent with the results of Zivick et al. (2018), who find little evidence of rotation in the SMC, which also supports the direct collision model.

\subsection{Field $O B$ Kinematics and Runaway Stars}

As noted in Section 1, non-compact binaries that are runaway systems must result from the dynamical ejection mechanism. In addition to SB2s, non-compact binaries also can be identified as eclipsing binaries (EBs), which are given in the Optical Gravitational Lensing Experiment (OGLE)-III EB catalog for the SMC (Pawlak et al. 2016). Of the 315 stars, only 295 are covered by the OGLE-III survey, as the easternmost Wing region is excluded. Furthermore, it is also possible to identify binaries with a compact remnant as high-mass X-ray binaries (HMXBs), which are compiled by Haberl \& Sturm (2016). Runaway HMXBs result from the SN acceleration mechanism. Stars found to be SB2, EB, and/or HMXB are indicated in Table 1.

In Table 2, we list the numbers of stars in each population, along with the mean and median $v_{\perp}$ and standard deviations $\sigma$ for each binary population. The median Gaia errors in $\boldsymbol{v}_{\alpha}, \boldsymbol{v}_{\delta}$, and $v_{\perp}$ for the sample are 22,17 , and $28 \mathrm{~km} \mathrm{~s}^{-1}$, with standard deviations on the errors of 5,3 , and $6 \mathrm{~km} \mathrm{~s}^{-1}$, respectively; thus the errors are more than a factor of 2 below the respective observed $\sigma_{\alpha}, \sigma_{\delta}$, and $\sigma_{\perp}$. Therefore, these standard deviations reflect actual velocity dispersions convolved with the substantial errors. Figure 2 compares PM and RV for the 207 stars 
Table 1

Kinematic Data for RIOTS4 Field OB Stars

\begin{tabular}{|c|c|c|c|c|c|c|c|c|c|c|c|c|c|}
\hline $\mathrm{ID}^{\mathrm{a}}$ & Subgroup $^{b}$ & $\begin{array}{c}v_{\perp}{ }^{\mathrm{c}} \\
\left(\mathrm{km} \mathrm{s}^{-1}\right)\end{array}$ & $\begin{array}{c}v_{\text {loc }, \perp}{ }^{\mathrm{c}} \\
\left(\mathrm{km} \mathrm{s}^{-1}\right)\end{array}$ & Quality $^{\mathrm{d}}$ & $\begin{array}{c}\mathrm{RV}^{\mathrm{c}} \\
\left(\mathrm{km} \mathrm{s}^{-1}\right)\end{array}$ & $\begin{array}{c}v_{\mathrm{R} . \mathrm{A} .} \mathrm{e}^{\mathrm{e}} \\
\left(\mathrm{km} \mathrm{s}^{-1}\right)\end{array}$ & $\begin{array}{c}\operatorname{err}^{\mathrm{f}} \\
\left(\mathrm{km} \mathrm{s}^{-1}\right)\end{array}$ & $\begin{array}{c}v_{\text {decl. }}^{\mathrm{e}} \\
\left(\mathrm{km} \mathrm{s}^{-1}\right)\end{array}$ & $\begin{array}{c}\operatorname{err}^{\mathrm{f}} \\
\left(\mathrm{km} \mathrm{s}^{-1}\right)\end{array}$ & $\begin{array}{l}v_{\text {loc, R.A. }}{ }^{\mathrm{e}} \\
\left(\mathrm{km} \mathrm{s}^{-1}\right)\end{array}$ & $\begin{array}{c}\operatorname{err}^{\mathrm{f}} \\
\left(\mathrm{km} \mathrm{s}^{-1}\right)\end{array}$ & $\begin{array}{l}v_{\text {loc, decl. }}{ }^{\mathrm{e}} \\
\left(\mathrm{km} \mathrm{s}^{-1}\right)\end{array}$ & $\begin{array}{c}\operatorname{err}^{\mathrm{f}} \\
\left(\mathrm{km} \mathrm{s}^{-1}\right)\end{array}$ \\
\hline 107 & $-,-,-, \mathrm{B},-$ & 42 & 20 & $-, 0,0$ & $\cdots$ & 186 & 17 & -347 & 12 & 167 & 5 & -349 & 7 \\
\hline 1037 & $-,-,-, \mathrm{B},-$ & 145 & 99 & $-, 0,0$ & -43 & 85 & 24 & -300 & 18 & 166 & 4 & -356 & 4 \\
\hline 1600 & $-, \mathrm{E},-, \mathrm{B},-$ & 22 & 43 & $-, 0,0$ & -58 & 188 & 22 & -401 & 21 & 187 & 5 & -358 & 4 \\
\hline 1631 & $-,-,-, \mathrm{B},-$ & 87 & 51 & $-, 0,0$ & -33 & 190 & 17 & -298 & 15 & 175 & 2 & -347 & 7 \\
\hline 1830 &,,,--- B,- & 75 & 32 & $-, 0,0$ & $\ldots$ & 143 & 20 & -341 & 17 & 174 & 4 & -349 & 6 \\
\hline 2034 & $-,-,-, \mathrm{B},-$ & 47 & 21 & $-, 0,0$ & $\ldots$ & 186 & 21 & -339 & 15 & 174 & 2 & -357 & 5 \\
\hline 2093 &,,,--- B,- & 105 & 63 & $-, 0,0$ & $\ldots$ & 122 & 20 & -318 & 18 & 178 & 5 & -347 & 8 \\
\hline 3224 & $-,-,-, B,-$ & 39 & 9 & $-, 0,0$ & -51 & 169 & 15 & -368 & 15 & 169 & 3 & -359 & 4 \\
\hline 3459 & $-,-,-, \mathrm{B},-$ & 39 & 7 & $-, 0,0$ & 50 & 183 & 21 & -353 & 17 & 179 & 5 & -359 & 3 \\
\hline 3815 & $-,-,-, \mathrm{B},-$ & 181 & 147 & $-, 0,1$ & $\ldots$ & 182 & 21 & -204 & 20 & 181 & 4 & -351 & 3 \\
\hline
\end{tabular}

Notes.

${ }^{\text {a }}$ From Massey (2002).

b "E," "S," "X" indicate EB, SB2, and HMXB, respectively; "B," "W," "D" indicate Bar, Wing, and boundary stars, respectively; "m" indicates object in multi-epoch spectroscopic sample.

${ }^{\mathrm{c}}$ Final residual velocity relative to SMC and Wing systemic motion. The $v_{\mathrm{loc}, \perp}$ values are computed relative to local velocity fields (see the text). RV errors are typically $10 \mathrm{~km} \mathrm{~s}{ }^{-1}$.

d "a" indicates object meets $\sim 10 \%$ asymmetry criterion in R.A. versus decl.; the second and third values give the number of stars within 1 " and 1 ". 5 , respectively.

e Transverse velocity computed from proper motion, without geometric or systemic velocity corrections.

${ }^{\mathrm{f}}$ Measurement errors, not including systematic errors (see the text).

(This table is available in its entirety in machine-readable form.) 

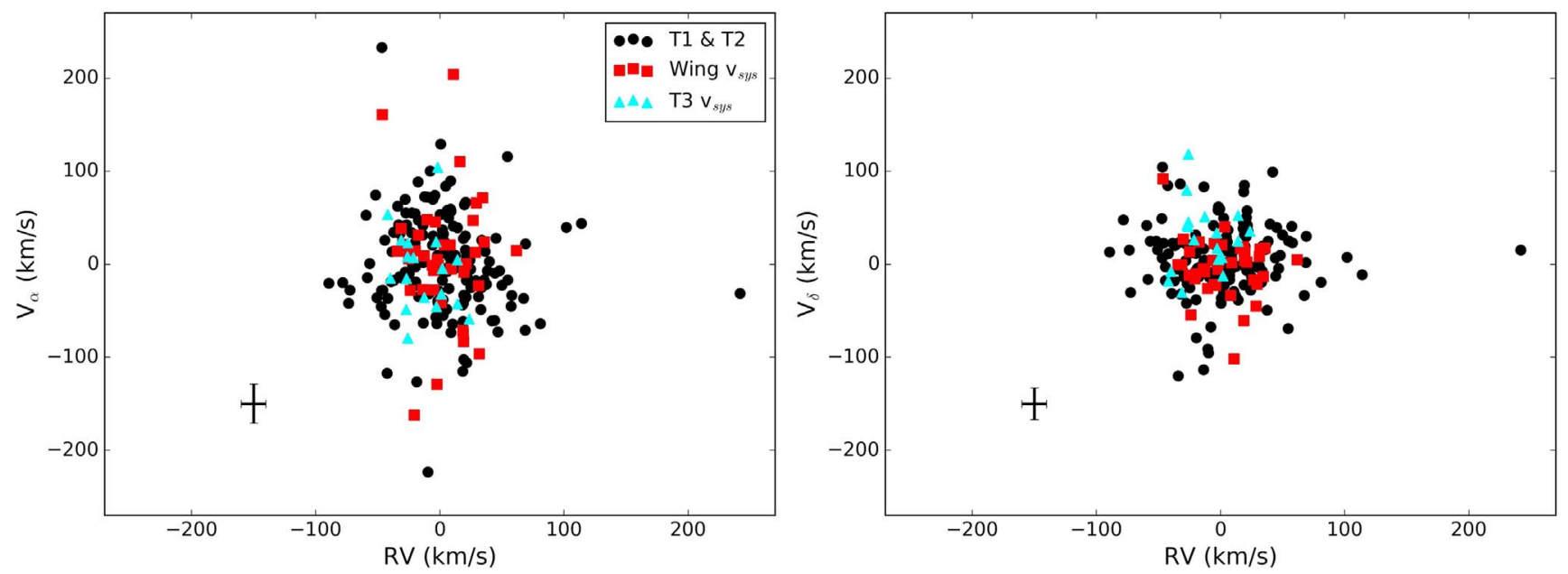

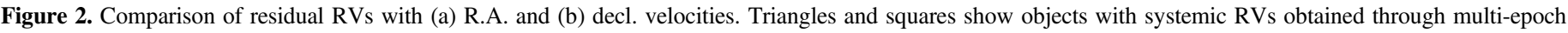
monitoring. The remainder of the sample are single-epoch RV measurements. Subsample labels are as in Figure 1.

Table 2

Kinematics of Binary SMC Field OB Stars

\begin{tabular}{lccccc}
\hline \hline & Unclassified $^{\mathrm{a}}$ & EB & SB2 & HMXB & Total \\
\hline Number & 267 & $16^{\mathrm{b}}$ & $10^{\mathrm{b}}$ & 15 & 304 \\
$\sigma_{\perp} / \mathrm{km} \mathrm{s}^{-1 \mathrm{c}}$ & 43 & 42 & 45 & 23 & 42 \\
$\sigma_{\alpha} / \mathrm{km} \mathrm{s}^{-1}$ & 56 & 52 & 47 & 32 & 55 \\
$\sigma_{\delta} / \mathrm{km} \mathrm{s}^{-1}$ & 37 & 33 & 42 & 26 & 37 \\
Median $\left(v_{\perp} / \mathrm{km} \mathrm{s}^{-1}\right)$ & 44 & 39 & 38 & 38 & 43 \\
Mean $\left(v_{\alpha} / \mathrm{km} \mathrm{s}^{-1}\right)$ & -3 & 16 & 16 & 1 & -1 \\
Mean $\left(v_{\delta} / \mathrm{km} \mathrm{s}^{-1}\right)$ & 7 & 8 & -25 & 9 & 6 \\
\hline$\sigma_{\text {loc }, \perp} / \mathrm{km} \mathrm{s}^{-1 \mathrm{c}}$ & 42 & 51 & 34 & 21 & 41 \\
$\sigma_{\text {loc }, \alpha} / \mathrm{km} \mathrm{s}^{-1}$ & 53 & 50 & 42 & 31 & 52 \\
$\sigma_{\text {loc }, \delta} / \mathrm{km} \mathrm{s}^{-1}$ & 35 & 33 & 37 & 23 & 35 \\
Median $\left(v_{\text {loc, }, \perp} / \mathrm{km} \mathrm{s}^{-1}\right)$ & 39 & 29 & 50 & 31 & 39 \\
Mean $\left(v_{\text {loc }, \alpha} / \mathrm{km} \mathrm{s}^{-1}\right)$ & 10 & 11 & 33 & 17 & 10 \\
Mean $\left(v_{\text {loc }, \delta} / \mathrm{km} \mathrm{s}^{-1}\right)$ & 3 & 2 & -29 & -4 & 2 \\
\hline
\end{tabular}

Notes.

a Includes unidentified binaries.

$\mathrm{b}$ There are four stars identified as both EB and SB2.

${ }^{\mathrm{c}}$ Values for $\sigma_{\perp}$ and $\sigma_{\mathrm{loc}, \perp}$ are standard deviations from the median.

that also have RVs reported by Lamb et al. (2016), omitting the stars near the Wing-Bridge boundary. Stars shown by triangles and squares are, respectively, systemic RVs estimated from our multi-epoch monitoring surveys of the Bar (Table 3 of Lamb et al. 2016) and Wing region (ongoing). We caution that the single-epoch observations often include significant binary motions. Figure 2 confirms that the measured PMs and RVs are comparable. It is apparent that the PMs show a larger spread in R.A. than decl., which is due to the asymmetric Gaia errors (e.g., Gaia Collaboration et al. 2018). Thus, velocities, especially large ones, for any given star may not be real, but the kinematics of subsamples may be compared. We have also inspected the OGLE-III (Udalski et al. 2008) images available for 304 of our target stars to evaluate point-spread function (PSF) asymmetry and crowding, which can degrade the astrometry; stars that may be thus affected are flagged in Table 1. Measured $v_{\perp}$ have no apparent dependence on PSF asymmetry when flagging those with $\gtrsim 10 \%$ variation in R.A. versus decl. But whereas $\sim 10 \%$ of all targets have neighbors within 1".5, targets having $v_{\perp}>1 \sigma_{\perp}$ are much more likely ( $24 \%, 10$ out of 41$)$ to have such close neighbors. Thus we caution that stars with $v_{\perp} \gtrsim 200 \mathrm{~km} \mathrm{~s}^{-1}$ are likely dominated by spurious values (e.g., Platais et al. 2018).

Figure 3(a) shows $v_{\perp}$ distributions for the 304 stars, which exclude those near the Wing-Bar boundary. We also show the contribution of each binary population. The peak of the $v_{\perp}$ distribution occurs at the value corresponding to the median error of $28 \mathrm{~km} \mathrm{~s}^{-1}$, reflecting the Gaia detection limit at $G<15$. The median $v_{\perp}$ of $44 \mathrm{~km} \mathrm{~s}^{-1}$ is therefore significant, occurring in the runaway velocity regime, as the typical velocity dispersion in $\mathrm{OB}$ associations is $\sim 5 \mathrm{~km} \mathrm{~s}^{-1}$ (e.g., Mel'nik \& Dambis 2017). Because half of the sample has $v_{\perp}$ greater than the median value, which in turn is larger than typical bound velocity dispersions, this therefore implies that well over half of the sample corresponds to unbound runaways, as many unbound stars also occur at velocities below the median (e.g., Renzo et al. 2018).

The population of "unclassified" stars in Table 2 simply refers to the remainder of the sample excluded by the other categories, and therefore includes any unidentified binaries. Thus, the EB, SB2, and HMXB populations are lower limits on the true numbers of non-compact and compact binary systems. Table 2 identifies 22 non-compact systems and 15 compact ones. We caution that the field includes a likely substantial population of non-runaway stars that formed in situ (e.g., Oey et al. 2013); analysis of the binary frequencies will be presented in a future work. Figure 3 and Table 2 show that the kinematics of the EB and SB2 populations are generally consistent with those of the total population, showing similar transverse velocity dispersions. In contrast, the spreads for the HMXBs are much lower, with the non-compact binaries having values about 50\% larger than for the HMXBs. In fact, none of the HMXBs have $v_{\perp}>1 \sigma$ from the median of the total sample (Figure 3(a)). We caution that one SB2 with velocity $>1 \sigma$, star 76253 , has another star within 1 !'5 to the north, which may affect the Gaia astrometry (Table 1).

The above kinematics are derived from only two assumed systemic components, Wing and Bar, as described in Section 2. As there may be additional, higher-order systemic motions, we also examine the PMs of our sample stars relative to their local velocity fields. We use the Gaia PMs of stars from the Massey (2002) catalog of OB stars within a $5^{\prime}(90 \mathrm{pc}$ ) radius of the 

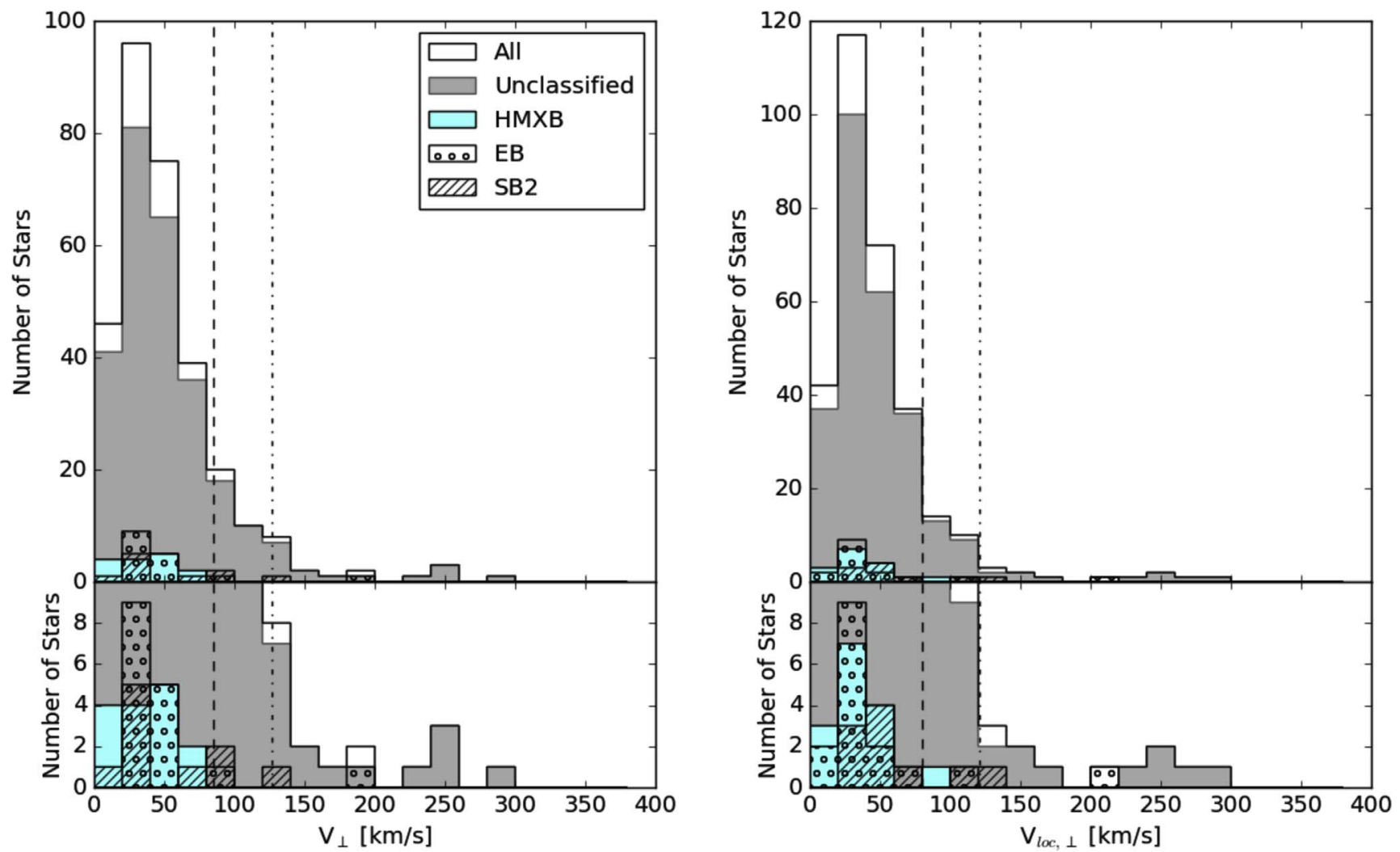

Figure 3. Distribution of $(a) v_{\perp}$ and $(b) v_{\mathrm{loc}, \perp}$. EB, SB2, and HMXB populations are shown as indicated. The vertical lines correspond to 1- $\sigma$ and 2- $\sigma$ velocities from the medians, using values in Table 2 . The lower insets show the $y$-axis zoomed for clarity.

target star to obtain the mean local velocity of the young population. We fitted the local PM distributions in R.A. and decl. with gaussians having $\sigma=45 \mathrm{~km} \mathrm{~s}^{-1}$ and $55 \mathrm{~km} \mathrm{~s}^{-1}$, respectively; these are the mean values for the Bar. The local transverse velocities $v_{\text {loc }}$ obtained in this way are given in Table 1. Figure 3(b) and Table 2 show the resulting residual PM kinematics. We see a similar pattern as before, with the HMXBs again showing smaller standard deviations $\sigma_{\text {loc }}$ when measured relative to the local fields.

We caution that K-S tests show that the difference between the $v_{\perp}$ distributions of the binary populations is not statistically significant. However, our sample likely contains a substantial, perhaps even dominant, contribution from non-runaway field stars that formed in situ (Oey et al. 2013; Lamb et al. 2016), which will significantly dilute the non-compact binary population in our sample at the lowest velocities. The fact that our HMXBs have smaller velocity dispersions than non-compact binaries is consistent with the expectation that bound compact binaries represent systems with less energetic SN kicks that failed to unbind the components. Moreover, dynamical ejections from dense clusters can accelerate runaways to higher velocities than the SN mechanism, because cluster acceleration can leverage the gravitational energy from multiple stars. Models by, for example, Brandt \& Podsiadlowski (1995) and Renzo et al. (2018) show that HMXBs have runaway velocities $<100 \mathrm{~km} \mathrm{~s}^{-1}$, and typically half of that value, depending on the assumed kick velocities and pre-SN orbital parameters. In contrast, Perets \& Šubr (2012) found that dynamically ejected runaways from clusters having masses on the order of $10^{4} \mathrm{M}_{\odot}$ can reach $200 \mathrm{~km} \mathrm{~s}^{-1}$, including significant fractions of binaries.

Despite contamination from non-runaways systems, the noncompact binaries show velocity distributions that are not only larger than for the HMXBs, but also similar to that for unclassified field OB stars (Table 2). The latter include singlestar runaways from both mechanisms, so this suggests that dynamically ejected objects dominate over in situ field stars in the SMC. Furthermore, Renzo et al. (2018) predicted that $\sim 14 \%$ of post-SN binaries fail to disrupt, of which some fraction are observed as HMXBs. They also expect $\sim 3 \%$ of post-SN binaries to generate single runaways faster than $30 \mathrm{~km} \mathrm{~s}^{-1}$. These estimates have large uncertainties, so we might expect roughly similar numbers of these two groups. However, there are only 15 HMXBs, whereas roughly half (134) of unclassified stars are fast runaways (median $v_{\perp}=44 \mathrm{~km} \mathrm{~s}^{-1}$ ). While these numbers are subject to various biases, the large disparity does suggest that dynamical ejections likely dominate. We will examine additional properties, including frequencies, masses, and rotation of these field OB runaways in future work.

We thank Roeland van der Marel for the use of his geometric correction code, and Michal K. Szymanski for help using OGLE data in preparatory work for this study. M.S.O. appreciates helpful discussions and generous hospitality from Kaitlin Kratter and the University of Arizona in hosting an extended visit supporting this collaboration. We thank the anonymous referee for helpful comments and suggestions. This work was funded by the National Science Foundation, grant AST-1514838 to MSO; and the University of Michigan. M.M. acknowledges support from NASA's Einstein Postdoctoral Fellowship program PF5-160139 and NASA ATP grant 17-ATP17-0070; N.K. is supported by NSF CAREER award AST-1455260. This research made use of Astropy, a community-developed core Python package for Astronomy.

Facilities: Gaia, Magellan. 


\section{ORCID iDs}

M. S. Oey (i) https://orcid.org/0000-0002-5808-1320

N. Castro ๑1 https://orcid.org/0000-0003-0521-473X

P. Zivick (1) https://orcid.org/0000-0001-9409-3911

N. Kallivayalil (i) https://orcid.org/0000-0002-3204-1742

D. J. Lennon (1) https://orcid.org/0000-0003-3063-4867

\section{References}

Battinelli, P. 1991, A\&A, 244, 69

Besla, G., Kallivayalil, N., Hernquist, L., et al. 2012, MNRAS, 421, 2109

Blaauw, A. 1961, BAN, 15, 265

Brandt, N., \& Podsiadlowski, P. 1995, MNRAS, 274, 461

Brück, M. T. 1978, A\&A, 68, 181

Cioni, M.-R. L., van der Marel, R. P., Loup, C., \& Habing, H. J. 2000, A\&A, 359,601

Clarke, C. J., \& Pringle, J. E. 1992, MNRAS, 255, 423

de Wit, W. J., Testi, L., Palla, F., \& Zinnecker, H. 2005, A\&A, 437, 247

Dobbie, P. D., Cole, A. A., Subramaniam, A., \& Keller, S. 2014, MNRAS, 442, 1663

Gaia Collaboration, Lindegren, L., Hernández, J., et al. 2018, A\&A, 616, A2 Gvaramadze, V. V., Pflamm-Altenburg, J., \& Kroupa, P. 2011, A\&A, 525, A17

Haberl, F., \& Sturm, R. 2016, A\&A, 586, A81

Hills, J. G. 1980, ApJ, 235, 986

Hoogerwerf, R., de Bruijne, J. H. J., \& de Zeeuw, P. T. 2000, ApJL, 544, L133

Lamb, J. B., Oey, M. S., Segura-Cox, D. M., et al. 2016, ApJ, 817, 113

Leonard, P. J. T., \& Duncan, M. J. 1988, AJ, 96, 222
Leonard, P. J. T., \& Duncan, M. J. 1990, AJ, 99, 608

Mason, B. D., Hartkopf, W. I., Gies, D. R., Henry, T. J., \& Helsel, J. W. 2009, AJ, 137, 3358

Massey, P. 2002, ApJ, 141, 81

Mel'nik, A. M., \& Dambis, A. K. 2017, MNRAS, 472, 3887

Moe, M., \& Di Stefano, R. 2015, ApJ, 810, 61

Moffat, A. F. J., Marchenko, S. V., Seggewiss, W., et al. 1998, A\&A, 331, 949

Oey, M. S., King, N. L., \& Parker, J. W. 2004, AJ, 127, 1632

Oey, M. S., Lamb, J. B., Kushner, C. T., Pellegrini, E. W., \& Graus, A. S. 2013, ApJ, 768, 66

Pawlak, M., Soszyński, I., Udalski, A., et al. 2016, AcA, 66, 421

Perets, H. B., \& Subr, L. 2012, ApJ, 751, 133

Pflamm-Altenburg, J., \& Kroupa, P. 2010, MNRAS, 404, 1564

Philp, C. J., Evans, C. R., Leonard, P. J. T., \& Frail, D. A. 1996, AJ, 111, 1220

Piatek, S., Pryor, C., \& Olszewski, E. W. 2008, AJ, 135, 1024

Platais, I., Lennon, D. J., van der Marel, R. P., et al. 2018, AJ, 156, 98

Portegies Zwart, S. F. 2000, ApJ, 544, 437

Poveda, A., Ruiz, J., \& Allen, C. 1967, BOTT, 4, 86

Renzo, M., Zapartas, E., de Mink, S. E., et al. 2018, A\&A, submitted (arXiv:1804.09164)

Ripepi, V., Cioni, M.-R. L., Moretti, M. I., et al. 2017, MNRAS, 472, 808

Sana, H., De Mink, S. E., De Koter, A., et al. 2012, Sci, 337, 444

Sayer, R. W., Nice, D. J., \& Kaspi, V. M. 1996, ApJ, 461, 357

Smith, R. C., Points, S. D., Chu, Y.-H., et al. 2005, BAAS, 37, 1200

Stanimirović, S., Staveley-Smith, L., \& Jones, P. A. 2004, ApJ, 604, 176

Udalski, A., Soszyński, I., Szymański, M. K., \& Al., E. 2008, AcA, 58, 329

van der Marel, R. P., Alves, D. R., Hardy, E., \& Suntzeff, N. B. 2002, AJ, 124, 2639

Zivick, P., Kallivayalil, N., van der Marel, R. P., et al. 2018, ApJ, 864, 55 